THE AsTROPHYSICAL JoURNAL, 517:650-660, 1999 June 1

(c) 1999. The American Astronomical Society. All rights reserved. Printed in U.S.A.

\title{
DETAILED SURFACE PHOTOMETRY OF DWARF ELLIPTICAL AND DWARF S0 GALAXIES IN THE VIRGO CLUSTER
}

\author{
Barbara S. Ryden, ${ }^{1,2}$ Donald M. Terndrup, ${ }^{2}$ and Richard W. Pogge \\ Department of Astronomy, The Ohio State University, 174 W. 18th Avenue, Columbus, OH 43210; ryden@astronomy.ohio-state.edu, \\ terndrup@astronomy.ohio-state.edu,pogge@astronomy.ohio-state.edu \\ AND \\ TOD R. LAUER \\ Kitt Peak National Observatory, NOAO, P.O. Box 26732, Tucson, AZ 85716; lauer@noao.edu \\ Received 1996 September 16; accepted 1999 January 4
}

\begin{abstract}
We analyze new $V$-band images of 14 dwarf S0 galaxies and 10 dwarf elliptical galaxies in the Virgo Cluster, in combination with $R$-band images of 70 dwarf elliptical galaxies from an earlier paper. We compute the intensity-weighted mean ellipticity, the mean deviations from elliptical isophotes, and a newly defined parameter to measure isophotal twists. We also fit each major-axis profile to a power law $\Sigma(a) \propto \exp \left[-\left(a / a_{s}\right)^{n}\right]$, where $n$ is allowed to vary. Consistent with other studies of the Virgo dwarf ellipticals, we find that the profile shapes for the entire sample is strongly peaked near $n=1$ (exponential profiles) and that no galaxies have $n=1 / 4$ (de Vaucouleurs profile). The faintest galaxies all have nearly exponential profiles, while the brighter ones on average have $n<1$. The correlation between ellipticity and the boxy/disky parameter is similar to that of large elliptical galaxies, suggesting that dwarfs may also be divided into two groups with differing internal dynamics. The Virgo dEs also show a greater degree of isophotal twisting than more luminous elliptical galaxies. There does not seem to be any combination of parameters from the surface photometry that statistically correlates with the $\mathrm{dE} / \mathrm{dS} 0$ designation: in particular, the dS0 galaxies do not, on average, have more pointed (disky) isophotes than the dEs.
\end{abstract}

Subject heading: galaxies: clusters: individual (Virgo) — galaxies: elliptical and lenticular, cD galaxies: photometry - galaxies: structure

\section{INTRODUCTION}

As is the case for their larger cousins, surface photometry of dwarf elliptical (dE) galaxies can provide some constraints on their origin, their three-dimensional shapes, and the importance of rotation versus velocity anisotropy (e.g., Kormendy \& Djorgovski 1989; Kormendy \& Bender 1996). Photometric studies are also typically the only way to study the $\mathrm{dE}$ galaxies, since they are almost always too faint for spectroscopic work to constrain the stellar dynamics of these systems.

In this paper, we continue our work on the $\mathrm{dE}$ galaxies of the Virgo Cluster. In our first paper (Ryden \& Terndrup 1994, hereafter Paper I), we presented $R$-band photometry of $70 \mathrm{dEs}$ and extensively discussed the distribution of apparent flattenings. In this work, we obtained $V$-band photometry of an additional $10 \mathrm{dEs}$, and observed 14 (nearly all) of the galaxies classified as possible dwarf S0 (dS0) systems in the Virgo Cluster Catalog (Binggeli, Sandage, \& Tammann 1985; hereafter VCC). The aims of this paper are to investigate the combined sample using several measures of the brightness profile and isophote shapes, in particular to extend our comparison of dwarf ellipticals with the more luminous elliptical galaxies.

A considerable amount is known about the properties of $\mathrm{dE}$ galaxies and how they differ from "ordinary" elliptical (E) galaxies. In contrast to Es, the $\mathrm{dE}$ galaxies have a lower central surface brightness (Reaves 1956, 1983; Binggeli et al. 1985), surface brightness profiles that are more nearly exponential than $r^{1 / 4}$ profiles (Caldwell 1983; Binggeli, Sandage,

\footnotetext{
${ }^{1}$ National Science Foundation Young Investigator.

${ }^{2}$ Visiting Observer, Lowell Observatory, Flagstaff, Arizona.
}

\& Tarenghi 1984; Ichikawa, Wakamatsu, \& Okamura 1986; Impey, Bothun, \& Malin 1988; Binggeli \& Cameron 1991; James 1991), and a markedly flatter distribution of projected ellipticity (Paper I, but see Binggeli \& Popescu 1995).

Much less is known about the dwarf S0 (dS0) galaxies in the Virgo Cluster. To see whether these have a more pronounced bulge/disk substructure than the dEs, we measured the radial brightness gradient in our combined sample, and measured the disky or boxy departures from elliptical isophotes. The latter quantities have been shown to indicate that some types of ellipticals are rotating and show disky isophote distortions, while others are slowly rotating and have boxy isophotes (Kormendy \& Bender 1996).

This paper is organized as follows. In $\S 2$, we present a discussion of our new observations and data reduction techniques, which were applied uniformly both to our new data and to those presented in Paper I. Section 3 presents the parameters we obtained to describe the surface photometry of the combined sample, and an analysis of these parameters. We close $(\S 4)$ with some final remarks about the nature of dwarf galaxies.

\section{OBSERVATIONS AND DATA REDUCTION}

We obtained $V$-band images of 14 dS0 galaxies and a further $10 \mathrm{dE}$ galaxies at the $1.8 \mathrm{~m}$ Perkins telescope ${ }^{3}$ over the period 1994 March 9-14 UT. We used the Ohio State Imaging Fabry-Perot System (IFPS), which is described in

\footnotetext{
${ }^{3}$ The Perkins Telescope is owned by Ohio Wesleyan University and was (until 1998 July) jointly operated by Lowell Observatory and Ohio State University.
} 
detail by Pogge et al. (1995). The detector was the Lowell Observatory Texas Instruments CCD $(800 \times 800$ pixels $)$, which in combination with the IFPS yields an unvignetted field of view of 5'.6 east-west and 6'.0 north-south at a scale of 0.49 pixel $^{-1}$. Table 1 lists the newly observed galaxies, the morphological designation of each galaxy from the VCC, and the number of exposures we obtained. The observing conditions were usually nonphotometric with varying amounts of thin cirrus. All exposures were $900 \mathrm{~s}$ in duration.

All reduction and analysis of the images was carried out using the Ohio State implementation of the Lick Observatory VISTA package (version 4.2). The raw images were reduced using a standard CCD reduction pipeline that applied a one-dimensional bias correction to each image using the signal in the overscan columns, followed by a flat-field correction derived from observations of an illuminated dome screen. The TI detector we used has no significant two-dimensional bias structure, so no "zero" correction is required. An additional reduction step was necessitated by the nonuniform illumination of the dome screen by a newly installed lamp system that resulted in unacceptably strong $( \pm 10 \%)$ variations in the flat field response. We corrected this by combining selected image frames into a "superflat" that was used to correct the pipeline images. The superflat produced images that were flat to about $\pm 0.5 \% \mathrm{rms}$ across the detector, with the exception of a localized $(100 \times 100$ pixel $)$ low-level $(\sim 1 \%)$ systematic flat-field error due to incomplete removal of objects in the superflat; this localized error causes minor distortion of the outermost isophotes of a few of our galaxies, although this does not affect our subsequent analysis. Many of the galaxies were observed in pairs of exposures; after pipeline and superflat reduction, the images were registered using shifts computed from measurements of field stars and added

TABLE 1

1994 OBSERVATIONS

\begin{tabular}{|c|c|c|}
\hline VCC & Class & Exposures \\
\hline $218 \ldots \ldots$ & $\mathrm{dSO}(8), \mathrm{N}:$ & $2^{\mathrm{a}}$ \\
\hline $275 \ldots \ldots$ & $\mathrm{dSO}(6)$ & 1 \\
\hline $389 \ldots \ldots$ & $\mathrm{dSO}(4), \mathrm{N}$ & 2 \\
\hline $510 \ldots \ldots$ & $\mathrm{dE} 3, \mathrm{~N}$ & 1 \\
\hline $751 \ldots \ldots$ & dS0 & 2 \\
\hline $781 \ldots \ldots$ & $\mathrm{dSO}_{3}(5), \mathrm{N}:$ & 2 \\
\hline $794 \ldots \ldots$ & dSO(8) pec: & 2 \\
\hline $951 \ldots \ldots$ & $\mathrm{dSO}(2), \mathrm{N}$ & 2 \\
\hline $990 \ldots \ldots$. & $\mathrm{dE} 4, \mathrm{~N}$ & 1 \\
\hline $1010 \ldots \ldots$ & $\mathrm{dS} 0(5), \mathrm{N}$ & 2 \\
\hline $1167 \ldots \ldots$ & $\mathrm{dE} 0, \mathrm{~N}$ & 1 \\
\hline $1308 \ldots \ldots$ & dE6,N & 1 \\
\hline $1333 \ldots \ldots$ & $\mathrm{dE} 0, \mathrm{~N}$ & 2 \\
\hline $1334 \ldots \ldots$ & $\mathrm{dSO}_{3}(8) ?$ & 2 \\
\hline $1386 \ldots \ldots$ & $\mathrm{dE} 3, \mathrm{~N}$ & 1 \\
\hline $1392 \ldots \ldots$ & dSB0(3), N & 2 \\
\hline $1684 \ldots \ldots$ & $\mathrm{dSO}(8):$ & 2 \\
\hline $1717 \ldots \ldots$ & dE7 & 1 \\
\hline $1779 \ldots \ldots$ & dSO(6): & 2 \\
\hline $1861 \ldots \ldots$ & $\mathrm{dE} 0, \mathrm{~N}$ & 1 \\
\hline $1876 \ldots \ldots$ & $\mathrm{dE} 5, \mathrm{~N}$ & 1 \\
\hline $1921 \ldots \ldots$ & $\mathrm{dSO}(8)$ & 2 \\
\hline $1936 \ldots \ldots$ & $\mathrm{dS} 0(0): \mathrm{N}$ & 1 \\
\hline $2019 \ldots \ldots$ & $\mathrm{dE} 4, \mathrm{~N}$ & 1 \\
\hline
\end{tabular}

${ }^{a}$ All exposures were $900 \mathrm{~s}$ in the $V$ band. together. Cosmic rays were removed by hand using an interactive surgical median-filtering routine. In a few cases, strongly saturated bright stars from previous images left residual charge patterns that persisted to the next exposure, and so for two galaxies we could use only one of the images in the pair.

\section{ANALYSIS OF THE SURFACE PHOTOMETRY}

We then combined the images from our 1994 observations with the $R$-band images of $70 \mathrm{dE}$ galaxies from the run in 1993 (Paper I), and analyzed both data sets in a uniform fashion (in the first paper, we derived profile shapes and mean axial ratios, but here we also measure isophotal twisting and departures of the isophotes from ellipses as described below).

We measured the surface brightness profiles of each galaxy with the VISTA routine PROFILE (Lauer 1985). Each galaxy was modeled as a series of concentric ellipses, yielding for each semimajor axis length $a$ the surface brightness $\Sigma(a)$ and the axial ratio $q \equiv b / a$, where $b$ is the length of the semiminor axis. The center of each ellipse was held fixed at the location of the intensity centroid in a small box (typically $15 \times 15$ pixels in size) centered on the central intensity maximum of each galaxy. Before measurement, stellar images that overlapped the galaxy images or that were nearby were eliminated by PSF subtraction using the version of DAOPHOT (Stetson 1987) installed as part of the VISTA package (this differs from the procedure in Paper I). The sky level on each frame was determined by computing the mean value of the modal sky in four to six regions surrounding the target galaxy; typically the error in the sky level as judged by the rms scatter in the model sky values was about $0.2 \%$ for the 1994 observations and $0.3 \%$ for the images from Paper I. The major-axis profiles for the 1994 data are plotted in Figure 1. See Paper I for representative plots of our earlier data.

\subsection{Ellipticity and Isophote Shapes}

The first statistic we measured was the intensity-weighted mean ellipticity. Using a slightly different notation than in Paper I, we write the area between two adjacent isophotes at major-axis distances $a$ and $a+d a$ and with axial ratios $q \equiv b / a$ as

$$
d A=2 \pi q a d a\left(1+\frac{1}{2} \frac{d \ln q}{d \ln a}\right),
$$

where $d a \ll a$. The luminosity between these isophotes is then given by

$$
d L=\Sigma(a) d A,
$$

and the total luminosity contained out to a distance $a_{0}$ is

$$
L(a)=\int_{0}^{a_{0}} \Sigma(a) d A .
$$

The luminosity-weighted mean axis ratio $\langle q\rangle$ can then be defined as

$$
\langle q\rangle=\int q d L / \int d L,
$$

and the corresponding mean ellipticity as $\langle\epsilon\rangle=1-\langle q\rangle$. In practice, the integrals in the above equation are carried out from an inner radius $a_{i}>0$ to reduce the effects of seeing in the centermost isophotes, to an fiducial radius $a_{0}$ 

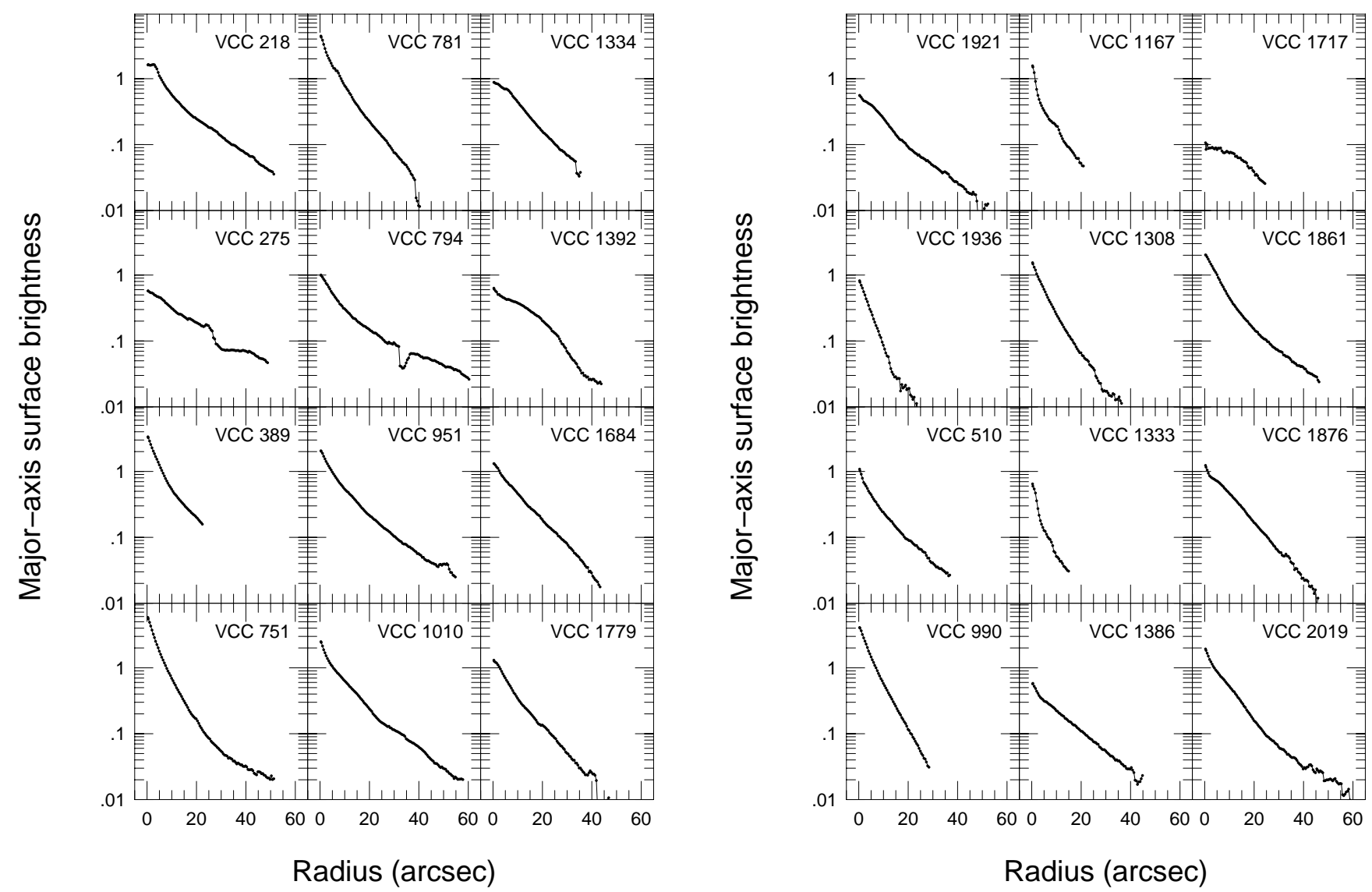

FIG. 1.-Surface brightness profiles for the 1994 data. The major-axis brightness profile for each galaxy is shown, where the units are relative to the local sky value. The galaxies are ordered by VCC number in two groups: the dS0 galaxies first, followed by the dE galaxies (see Table 1). Errors in the surface brightness are typically $1 \%$ in the inner part of the profile, rising to $20 \%$ for the outermost points.

where the errors in the surface brightness become large. For the combined data sets, we computed $\langle\epsilon\rangle$ using $a_{i}=5^{\prime \prime}$ and set $a_{0}$ to be the distance at which the intensity profile $\Sigma\left(a_{0}\right)$ fell to 180 counts. This outer level corresponds to $\approx 5 \%$ of the mean sky level for the data from both years. The computed values of $\langle\epsilon\rangle$ are insensitive to the choice of $a_{0}$ since typically the surface brightness falls off exponentially with radius (below).

The values of $\langle\epsilon\rangle$ for each galaxy are displayed in Table 2. The first column of that table lists the galaxy number from the VCC, while the second and third columns show, respectively, the galaxy classification from the VCC (simplified to dS0/dE and to show the presence of nuclei) and the year of observation. The next three columns show, respectively, the outer fiducial radius $a_{0}$ in arcseconds, $\langle\epsilon\rangle$, and the value of the ellipticity $\epsilon\left(a_{0}\right)$ at the fiducial radius.

Some of our galaxies were members of overlapping galaxy pairs, had a very bright star nearby or overlapping the galaxy image, or were crossed by bleeding along columns from a saturated image at more distant parts of the chip. Following the techniques in Paper I, we estimated $\langle\epsilon\rangle$ for these galaxies by inspection of contour maps of the isophotes; these galaxies are noted in the last column of Table 2. There were a few galaxies with the value $\langle\epsilon\rangle$ estimated this way in Paper I, but for which we could obtain good isophote fits this time, typically because the starsubtraction technique was significantly more accurate than our previous method.
Figure 3 shows the intensity-weighted mean ellipticity $\langle\epsilon\rangle$ against the ellipticity of the outer isophote $\epsilon\left(a_{0}\right)$. In this and many of the following plots, values for dS0 galaxies are shown as filled triangles, nonnucleated dEs as open circles, and nucleated dEs as open circles with a cross superimposed. The solid line denotes equality, while the error bar shows the mean uncertainty in the two quantities. The scatter about this line is only slightly larger than the average error of measurement. As with the dE galaxies, the $\mathrm{dS} 0$ s are not on average flatter in their outer profiles than they are close to their nuclei, as might be expected if the $\mathrm{dSO}$ s have a pronounced bulge/disk structure.

We also determined the departures of each isophote from an ellipse using the formulation of Carter (1978), in which the intensity is written as a Fourier expansion around the best-fitting elliptical isophote in the form

$$
I(\theta)=I_{0}+\sum_{n \geq 3}\left(A_{n} \cos n \theta+B_{n} \sin n \theta\right)
$$

where $\theta$ is the position angle measured from the major axis of the ellipse, and $I_{0}=\Sigma(\mathrm{a})$ is the surface brightness at each a. (We measured the values of $A_{n}$ for only a small subset of the images in Paper I.) The luminosity deviations $A_{n}$ and $B_{n}$ at a semimajor axis $a$ can be converted into fractional radial deviations $a_{n} / a$ and $b_{n} / a$ through the relations

$$
\begin{aligned}
& a_{n} / a=-A_{n}\left[a\left(d I_{0} / d a\right)\right], \\
& b_{n} / a=-B_{n}\left[a\left(d I_{0} / d a\right)\right] .
\end{aligned}
$$


TABLE 2

Results of the Surface Photometry

\begin{tabular}{|c|c|c|c|c|c|c|c|c|c|c|}
\hline $\mathrm{VCC}$ & Class & Year & $a_{0}(\operatorname{arcsec})$ & $\langle\epsilon\rangle$ & $\epsilon\left(a_{0}\right)$ & $\left\langle a_{4} / a\right\rangle$ & $\sigma\left(a_{4} / a\right)$ & $T$ & $n$ & $\sigma(n)$ \\
\hline $128 \ldots \ldots$. & $\mathrm{dE}$ & 93 & 31.6 & 0.14 & 0.24 & $7.88 \mathrm{e}-03$ & $4.44 \mathrm{e}-03$ & $2.92 \mathrm{e}-02$ & 1.18 & 0.12 \\
\hline $218 \ldots \ldots \ldots$ & $\mathrm{dS} 0, \mathrm{~N}$ & 94 & 51.0 & 0.64 & 0.67 & $-8.71 \mathrm{e}-04$ & $2.58 \mathrm{e}-03$ & $7.03 \mathrm{e}-03$ & 0.62 & 0.07 \\
\hline $273 \ldots \ldots$ & $\mathrm{dE}, \mathrm{N}$ & 93 & 36.3 & 0.30 & 0.31 & $1.33 e-03$ & $2.64 \mathrm{e}-03$ & $2.93 \mathrm{e}-03$ & 0.94 & 0.18 \\
\hline $275 \ldots \ldots$ & dSO & 94 & 31.6 & 0.46 & 0.46 & $4.78 \mathrm{e}-02$ & $3.00 \mathrm{e}-03$ & $3.89 \mathrm{e}-03$ & 0.60 & 0.08 \\
\hline $319 \ldots \ldots$ & $\mathrm{dE}, \mathrm{N}$ & 93 & $\ldots$ & $0.04^{\mathrm{a}}$ & $\ldots$ & $\ldots$ & $\ldots$ & $\ldots$ & $\ldots$ & $\ldots$ \\
\hline $389 \ldots \ldots$ & $\mathrm{dS} 0, \mathrm{~N}$ & 94 & 22.4 & 0.20 & 0.24 & $2.48 \mathrm{e}-03$ & $1.01 \mathrm{e}-03$ & $3.19 \mathrm{e}-03$ & 0.26 & 0.01 \\
\hline $421 \ldots \ldots$ & $\mathrm{dE}$ & 93 & 20.1 & 0.18 & 0.22 & $9.35 \mathrm{e}-04$ & $9.76 \mathrm{e}-03$ & $4.11 \mathrm{e}-03$ & 0.56 & 0.22 \\
\hline $458 \ldots \ldots$. & $\mathrm{dE}$ & 93 & 30.8 & 0.37 & 0.37 & $-1.99 \mathrm{e}-03$ & $3.86 \mathrm{e}-03$ & $3.36 \mathrm{e}-03$ & 1.15 & 0.31 \\
\hline $510 \ldots \ldots$ & $\mathrm{dE}, \mathrm{N}$ & 94 & 22.5 & 0.17 & 0.16 & $-6.61 \mathrm{e}-03$ & $1.78 \mathrm{e}-03$ & $4.08 \mathrm{e}-03$ & 0.69 & 0.21 \\
\hline $543 \ldots \ldots$ & $\mathrm{dE}$ & 93 & 83.2 & 0.44 & 0.44 & $5.35 \mathrm{e}-03$ & $1.89 \mathrm{e}-03$ & $1.05 \mathrm{e}-02$ & 0.57 & 0.12 \\
\hline $545 \ldots \ldots$ & $\mathrm{dE}, \mathrm{N}$ & 93 & 40.3 & 0.22 & 0.20 & $1.71 \mathrm{e}-02$ & $7.25 \mathrm{e}-03$ & $1.60 \mathrm{e}-03$ & 0.79 & 0.04 \\
\hline $551 \ldots \ldots$ & $\mathrm{dE}$ & 93 & 37.6 & 0.27 & 0.27 & $2.08 \mathrm{e}-03$ & $5.42 \mathrm{e}-03$ & $1.79 \mathrm{e}-03$ & 1.24 & 0.15 \\
\hline $592 \ldots \ldots$. & $\mathrm{dE}, \mathrm{N}$ & 93 & 37.8 & 0.39 & 0.33 & $5.25 \mathrm{e}-03$ & $2.73 e-03$ & $4.23 \mathrm{e}-04$ & 0.91 & 0.12 \\
\hline $608 \ldots \ldots$. & $\mathrm{dE}, \mathrm{N}$ & 93 & 49.6 & 0.39 & 0.25 & $-3.05 \mathrm{e}-02$ & $4.10 \mathrm{e}-03$ & $7.66 \mathrm{e}-03$ & 1.43 & 0.11 \\
\hline $611 \ldots \ldots$ & $\mathrm{dE}$ & 93 & 44.1 & 0.39 & 0.47 & $-2.25 \mathrm{e}-02$ & $5.69 \mathrm{e}-03$ & $1.75 \mathrm{e}-03$ & 1.00 & 0.07 \\
\hline $622 \ldots \ldots$. & $\mathrm{dE}$ & 93 & 18.3 & 0.44 & 0.49 & $-2.73 \mathrm{e}-02$ & $1.20 \mathrm{e}-02$ & $5.51 \mathrm{e}-03$ & 1.59 & 0.42 \\
\hline $684 \ldots \ldots$ & $\mathrm{dE}, \mathrm{N}$ & 93 & 36.8 & 0.09 & 0.02 & $4.74 \mathrm{e}-03$ & $2.30 \mathrm{e}-03$ & $1.78 \mathrm{e}-03$ & 0.90 & 0.33 \\
\hline $711 \ldots \ldots$ & $\mathrm{dE}, \mathrm{N}$ & 93 & 34.9 & 0.16 & 0.04 & $-9.99 \mathrm{e}-03$ & $3.55 \mathrm{e}-03$ & $1.85 \mathrm{e}-03$ & 1.08 & 0.17 \\
\hline $745 \ldots \ldots$ & $\mathrm{dE}, \mathrm{N}$ & 93 & 63.2 & 0.36 & 0.45 & $2.38 \mathrm{e}-03$ & $2.20 \mathrm{e}-03$ & $1.95 \mathrm{e}-03$ & 0.73 & 0.14 \\
\hline $750 \ldots \ldots$ & $\mathrm{dE}, \mathrm{N}$ & 93 & $\ldots$ & 0.24 & 0.39 & $-5.54 \mathrm{e}-03$ & $1.52 \mathrm{e}-03$ & $\ldots$ & 0.69 & 0.12 \\
\hline $751 \ldots \ldots$ & dS0 & 94 & 34.0 & 0.34 & 0.24 & $1.84 \mathrm{e}-02$ & $2.47 \mathrm{e}-03$ & $4.83 \mathrm{e}-03$ & 0.27 & 0.08 \\
\hline $753 \ldots \ldots$ & $\mathrm{dE}, \mathrm{N}$ & 93 & 34.7 & 0.09 & 0.11 & $-4.06 \mathrm{e}-03$ & $5.63 e-03$ & $6.51 \mathrm{e}-02$ & 1.18 & 0.17 \\
\hline $781 \ldots \ldots$ & $\mathrm{dS} 0, \mathrm{~N}$ & 94 & 35.8 & 0.38 & 0.42 & $-1.20 \mathrm{e}-03$ & $3.45 \mathrm{e}-03$ & $2.38 \mathrm{e}-03$ & 0.69 & 0.05 \\
\hline $794 \ldots \ldots$. & dS0 & 94 & 56.5 & 0.53 & 0.71 & $5.81 \mathrm{e}-03$ & $3.12 \mathrm{e}-03$ & $2.47 \mathrm{e}-03$ & 0.22 & 0.05 \\
\hline $810 \ldots \ldots$ & $\mathrm{dE}, \mathrm{N}$ & 93 & 28.6 & 0.05 & 0.01 & $-1.78 \mathrm{e}-02$ & $4.55 \mathrm{e}-03$ & $1.81 \mathrm{e}-02$ & 1.24 & 0.17 \\
\hline $816 \ldots \ldots$ & $\mathrm{dE}, \mathrm{N}$ & 93 & $\ldots$ & $0.10^{\mathrm{a}}$ & $\ldots$ & $\ldots$ & $\ldots$ & $\ldots$ & $\ldots$ & $\ldots$ \\
\hline $823 \ldots \ldots$ & $\mathrm{dE}, \mathrm{N}$ & 93 & 38.6 & 0.08 & 0.07 & $\ldots$ & $\ldots$ & $9.27 \mathrm{e}-04$ & 0.31 & 0.06 \\
\hline $931 \ldots \ldots$. & $\mathrm{dE}, \mathrm{N}$ & 93 & 29.5 & 0.19 & 0.28 & $-9.47 \mathrm{e}-03$ & $1.21 \mathrm{e}-02$ & $5.67 \mathrm{e}-02$ & 1.89 & 0.01 \\
\hline $933 \ldots \ldots$ & $\mathrm{dE}, \mathrm{N}$ & 93 & 23.6 & 0.32 & 0.37 & $1.83 \mathrm{e}-02$ & $6.63 \mathrm{e}-03$ & $2.96 \mathrm{e}-03$ & 0.69 & 0.16 \\
\hline $951 \ldots \ldots$. & dSO & 94 & 47.2 & 0.26 & 0.30 & $1.17 \mathrm{e}-02$ & $2.83 \mathrm{e}-03$ & $8.62 \mathrm{e}-03$ & 0.61 & 0.05 \\
\hline $990 \ldots \ldots$. & $\mathrm{dE}, \mathrm{N}$ & 94 & 24.2 & 0.29 & 0.24 & $2.91 \mathrm{e}-02$ & $3.08 \mathrm{e}-03$ & $3.37 \mathrm{e}-04$ & 0.91 & 0.14 \\
\hline $991 \ldots \ldots$. & $\mathrm{dE}$ & 93 & $\ldots$ & $0.38^{\mathrm{a}}$ & $\ldots$ & $\ldots$ & $\ldots$ & $\ldots$ & $\ldots$ & $\ldots$ \\
\hline $1010 \ldots \ldots$ & $\mathrm{dS} 0, \mathrm{~N}$ & 94 & 48.1 & 0.42 & 0.38 & $-1.12 \mathrm{e}-03$ & $1.38 \mathrm{e}-03$ & $1.47 \mathrm{e}-02$ & 0.69 & 0.09 \\
\hline $1044 \ldots \ldots$ & $\mathrm{dE}, \mathrm{N}$ & 93 & $\ldots$ & $0.34^{\mathrm{a}}$ & $\ldots$ & $-3.74 \mathrm{e}-04$ & $3.98 \mathrm{e}-03$ & $\ldots$ & 1.08 & 0.17 \\
\hline $1065 \ldots \ldots$ & $\mathrm{dE}, \mathrm{N}$ & 93 & 29.1 & 0.10 & 0.16 & $2.54 \mathrm{e}-03$ & $3.42 \mathrm{e}-03$ & $1.04 \mathrm{e}-03$ & 1.10 & 0.17 \\
\hline $1073 \ldots \ldots$ & $\mathrm{dE}, \mathrm{N}$ & 93 & $\ldots$ & $0.28^{\mathrm{a}}$ & $\ldots$ & $\ldots$ & $\ldots$ & $\ldots$ & $\ldots$ & $\ldots$ \\
\hline $1087 \ldots \ldots$ & $\mathrm{dE}, \mathrm{N}$ & 93 & $\ldots$ & $0.25^{\mathrm{a}}$ & $\ldots$ & $\ldots$ & $\ldots$ & $\ldots$ & $\ldots$ & $\ldots$ \\
\hline $1104 \ldots \ldots$ & $\mathrm{dE}, \mathrm{N}$ & 93 & 53.3 & 0.27 & 0.33 & $2.30 \mathrm{e}-02$ & $2.51 \mathrm{e}-03$ & $1.40 \mathrm{e}-03$ & 0.73 & 0.12 \\
\hline $1122 \ldots \ldots$ & $\mathrm{dE}, \mathrm{N}$ & 93 & 79.8 & 0.52 & 0.64 & $1.75 \mathrm{e}-02$ & $3.37 \mathrm{e}-03$ & $3.66 \mathrm{e}-04$ & 0.57 & 0.06 \\
\hline $1167 \ldots \ldots$ & $\mathrm{dE}, \mathrm{N}$ & 94 & 16.9 & 0.09 & 0.03 & $-5.38 \mathrm{e}-03$ & $5.78 \mathrm{e}-03$ & $3.20 \mathrm{e}-02$ & 0.76 & 0.18 \\
\hline $1180 \ldots \ldots$ & $\mathrm{dE}$ & 93 & 34.9 & 0.22 & 0.22 & $3.38 \mathrm{e}-03$ & $5.02 \mathrm{e}-03$ & $2.84 \mathrm{e}-03$ & 1.16 & 0.16 \\
\hline $1223 \ldots \ldots$ & $\mathrm{dE}$ & 93 & 24.6 & 0.43 & 0.41 & $-3.87 \mathrm{e}-03$ & $1.05 \mathrm{e}-02$ & $3.09 \mathrm{e}-03$ & 0.89 & 0.69 \\
\hline $1240 \ldots \ldots$ & $\mathrm{dE}, \mathrm{N}$ & 93 & 27.9 & 0.41 & 0.48 & $2.73 \mathrm{e}-03$ & $5.58 \mathrm{e}-03$ & $9.52 \mathrm{e}-03$ & 0.93 & 0.17 \\
\hline $1264 \ldots \ldots$ & $\mathrm{dE}, \mathrm{N}$ & 93 & $\ldots$ & $0.22^{\mathrm{a}}$ & $\ldots$ & $\ldots$ & $\ldots$ & $\ldots$ & $\ldots$ & $\ldots$ \\
\hline $1308 \ldots \ldots$ & $\mathrm{dE}, \mathrm{N}$ & 94 & 20.1 & 0.26 & 0.41 & $1.12 \mathrm{e}-02$ & $2.91 \mathrm{e}-03$ & $4.37 \mathrm{e}-03$ & 0.83 & 0.41 \\
\hline $1333 \ldots \ldots$ & $\mathrm{dE}, \mathrm{N}$ & 94 & 13.8 & 0.21 & 0.09 & $3.76 \mathrm{e}-03$ & $1.13 \mathrm{e}-02$ & $1.26 \mathrm{e}-02$ & 0.57 & 0.37 \\
\hline $1334 \ldots \ldots$ & dS0 & 94 & 33.7 & 0.59 & 0.52 & $1.68 \mathrm{e}-02$ & $1.69 \mathrm{e}-03$ & $3.71 \mathrm{e}-03$ & 0.87 & 0.09 \\
\hline $1351 \ldots \ldots$ & $\mathrm{dE}$ & 93 & 54.7 & 0.33 & 0.29 & $-1.24 \mathrm{e}-02$ & $1.03 \mathrm{e}-02$ & $1.76 \mathrm{e}-02$ & $\ldots$ & $\ldots$ \\
\hline $1355 \ldots \ldots$ & $\mathrm{dE}, \mathrm{N}$ & 93 & 65.1 & 0.22 & 0.15 & $1.47 \mathrm{e}-02$ & $2.92 \mathrm{e}-03$ & $5.42 \mathrm{e}-03$ & 0.78 & 0.05 \\
\hline $1386 \ldots \ldots$ & $\mathrm{dE}, \mathrm{N}$ & 94 & 28.0 & 0.28 & 0.33 & $8.26 \mathrm{e}-03$ & $1.66 \mathrm{e}-03$ & $5.22 \mathrm{e}-03$ & 0.93 & 0.17 \\
\hline $1392 \ldots \ldots$ & dSO & 94 & 34.8 & 0.44 & 0.08 & $-4.61 \mathrm{e}-03$ & $1.97 \mathrm{e}-03$ & $2.29 \mathrm{e}-03$ & 2.04 & 0.08 \\
\hline $1407 \ldots \ldots$ & $\mathrm{dE}, \mathrm{N}$ & 93 & 56.8 & 0.15 & 0.15 & $5.44 \mathrm{e}-03$ & $1.15 \mathrm{e}-03$ & $4.35 \mathrm{e}-04$ & 0.48 & 0.05 \\
\hline $1431 \ldots \ldots$ & $\mathrm{dE}, \mathrm{N}$ & 93 & 57.8 & 0.04 & 0.01 & $1.38 \mathrm{e}-03$ & $1.73 \mathrm{e}-03$ & $2.33 \mathrm{e}-03$ & 0.67 & 0.12 \\
\hline $1432 \ldots \ldots$ & $\mathrm{dE}$ & 93 & 25.0 & 0.11 & 0.18 & $-6.76 \mathrm{e}-03$ & $6.45 \mathrm{e}-03$ & $3.52 \mathrm{e}-03$ & 1.75 & 0.43 \\
\hline $1446 \ldots \ldots$ & $\mathrm{dE}, \mathrm{N}$ & 93 & 40.7 & 0.09 & 0.08 & $-1.53 \mathrm{e}-03$ & $3.04 \mathrm{e}-03$ & $4.33 \mathrm{e}-03$ & 0.86 & 0.31 \\
\hline $1489 \ldots \ldots$ & $\mathrm{dE}, \mathrm{N}$ & 93 & 50.3 & 0.40 & 0.45 & $1.28 \mathrm{e}-02$ & $2.19 \mathrm{e}-03$ & $1.03 \mathrm{e}-03$ & 0.84 & 0.08 \\
\hline $1491 \ldots \ldots$ & $\mathrm{dE}, \mathrm{N}$ & 93 & 57.6 & 0.21 & 0.33 & 4.60e-04 & $1.35 \mathrm{e}-03$ & $5.27 \mathrm{e}-03$ & 0.33 & 0.29 \\
\hline $1503 \ldots \ldots$ & $\mathrm{dE}, \mathrm{N}$ & 93 & 54.7 & 0.15 & 0.20 & $-9.88 \mathrm{e}-03$ & $2.11 \mathrm{e}-03$ & $4.98 \mathrm{e}-03$ & 0.71 & 0.09 \\
\hline $1514 \ldots \ldots$ & $\mathrm{dE}, \mathrm{N}$ & 93 & 73.6 & 0.64 & 0.49 & $2.70 \mathrm{e}-02$ & $2.84 \mathrm{e}-03$ & $3.62 \mathrm{e}-03$ & 0.98 & 0.05 \\
\hline $1539 \ldots \ldots$ & $\mathrm{dE}, \mathrm{N}$ & 93 & 44.7 & 0.11 & 0.05 & $5.83 \mathrm{e}-03$ & $4.76 \mathrm{e}-03$ & $1.15 \mathrm{e}-02$ & 0.89 & 0.23 \\
\hline $1563 \ldots \ldots$ & $\mathrm{dE}, \mathrm{N}$ & 93 & 42.8 & 0.29 & 0.35 & $-6.28 \mathrm{e}-03$ & $2.96 \mathrm{e}-03$ & $7.87 \mathrm{e}-03$ & 1.30 & 0.25 \\
\hline $1577 \ldots \ldots$ & $\mathrm{dE}$ & 93 & 34.1 & 0.23 & 0.30 & $1.32 \mathrm{e}-02$ & $3.59 \mathrm{e}-03$ & $1.81 \mathrm{e}-04$ & 0.92 & 0.34 \\
\hline $1649 \ldots \ldots$ & $\mathrm{dE}, \mathrm{N}$ & 93 & 48.4 & 0.23 & 0.28 & $5.06 \mathrm{e}-03$ & $3.38 \mathrm{e}-03$ & $7.22 \mathrm{e}-04$ & 0.94 & 0.11 \\
\hline $1651 \ldots \ldots$ & $\mathrm{dE}$ & 93 & 8.7 & 0.41 & 0.15 & $7.52 \mathrm{e}-03$ & $3.52 \mathrm{e}-02$ & $1.85 \mathrm{e}-03$ & 0.41 & 0.11 \\
\hline $1669 \ldots \ldots$ & $\mathrm{dE}, \mathrm{N}$ & 93 & 50.3 & 0.54 & 0.58 & $1.64 \mathrm{e}-02$ & $2.65 \mathrm{e}-03$ & $2.89 \mathrm{e}-03$ & 1.47 & 0.20 \\
\hline
\end{tabular}


TABLE 2-Continued

\begin{tabular}{|c|c|c|c|c|c|c|c|c|c|c|}
\hline VCC & Class & Year & $a_{0}(\operatorname{arcsec})$ & $\langle\epsilon\rangle$ & $\epsilon\left(a_{0}\right)$ & $\left\langle a_{4} / a\right\rangle$ & $\sigma\left(a_{4} / a\right)$ & $T$ & $n$ & $\sigma(n)$ \\
\hline $1677 \ldots \ldots$ & $\mathrm{dE}, \mathrm{N}$ & 93 & 19.7 & 0.26 & 0.16 & $-2.74 \mathrm{e}-03$ & $5.80 \mathrm{e}-03$ & $4.74 \mathrm{e}-03$ & 1.89 & 0.06 \\
\hline $1683 \ldots \ldots$ & $\mathrm{dE}, \mathrm{N}$ & 93 & $\ldots$ & $0.10^{\mathrm{a}}$ & $\ldots$ & $\ldots$ & $\ldots$ & $\ldots$ & $\ldots$ & $\ldots$ \\
\hline $1684 \ldots \ldots$ & $\mathrm{dS} 0$ & 94 & 38.4 & 0.62 & 0.52 & $-4.49 \mathrm{e}-02$ & $5.68 \mathrm{e}-03$ & $8.93 \mathrm{e}-03$ & 0.98 & 0.07 \\
\hline $1689 \ldots \ldots$ & $\mathrm{dE}$ & 93 & 22.7 & 0.18 & 0.09 & $\ldots$ & $\ldots$ & $7.30 \mathrm{e}-02$ & 1.70 & 0.29 \\
\hline $1698 \ldots \ldots$ & $\mathrm{dE}$ & 93 & 46.1 & 0.50 & 0.35 & $-6.53 \mathrm{e}-03$ & $3.80 \mathrm{e}-03$ & $8.93 \mathrm{e}-04$ & 1.00 & 0.19 \\
\hline $1704 \ldots \ldots$ & $\mathrm{dE}$ & 93 & 41.3 & 0.46 & 0.47 & $1.13 \mathrm{e}-02$ & $3.10 \mathrm{e}-03$ & $1.01 \mathrm{e}-03$ & 1.43 & 0.20 \\
\hline $1717 \ldots \ldots$ & $\mathrm{dE}$ & 94 & 12.1 & 0.64 & 0.70 & $6.76 \mathrm{e}-03$ & $1.24 \mathrm{e}-02$ & $6.40 \mathrm{e}-03$ & 2.27 & 0.24 \\
\hline $1743 \ldots \ldots$ & $\mathrm{dE}$ & 93 & 52.9 & 0.52 & 0.40 & $-2.56 \mathrm{e}-02$ & $3.66 \mathrm{e}-03$ & $1.35 \mathrm{e}-04$ & 1.10 & 0.17 \\
\hline $1762 \ldots \ldots$ & $\mathrm{dE}$ & 93 & 39.6 & 0.50 & 0.54 & $4.18 \mathrm{e}-03$ & $2.97 \mathrm{e}-03$ & $3.56 \mathrm{e}-04$ & 0.62 & 0.20 \\
\hline $1767 \ldots \ldots$ & $\mathrm{dE}, \mathrm{N}$ & 93 & 36.3 & 0.29 & 0.36 & $9.90 \mathrm{e}-03$ & $3.86 \mathrm{e}-03$ & $5.73 \mathrm{e}-03$ & 1.18 & 0.22 \\
\hline $1779 \ldots \ldots$ & dS0 & 94 & 0.5 & 0.49 & 0.50 & $-2.03 \mathrm{e}-02$ & $3.64 \mathrm{e}-03$ & $3.06 \mathrm{e}-02$ & 0.83 & 0.07 \\
\hline $1803 \ldots \ldots$ & $\mathrm{dE}, \mathrm{N}$ & 93 & 32.7 & 0.04 & 0.19 & $-2.97 \mathrm{e}-03$ & $3.65 \mathrm{e}-03$ & $1.39 \mathrm{e}-02$ & 0.48 & 0.07 \\
\hline $1861 \ldots \ldots$ & $\mathrm{dE}, \mathrm{N}$ & 94 & 30.0 & 0.03 & 0.05 & $-8.67 \mathrm{e}-04$ & $1.36 \mathrm{e}-03$ & $1.97 \mathrm{e}-02$ & 0.37 & 0.14 \\
\hline $1876 \ldots . .$. & $\mathrm{dE}, \mathrm{N}$ & 94 & 27.8 & 0.47 & 0.49 & $1.36 \mathrm{e}-02$ & $1.75 \mathrm{e}-03$ & $1.76 \mathrm{e}-03$ & 0.99 & 0.31 \\
\hline $1886 \ldots . .$. & $\mathrm{dE}, \mathrm{N}$ & 93 & 61.3 & 0.39 & 0.43 & $1.37 \mathrm{e}-02$ & $2.23 \mathrm{e}-03$ & $1.69 \mathrm{e}-03$ & 1.04 & 0.09 \\
\hline $1919 \ldots \ldots$ & $\mathrm{dE}, \mathrm{N}$ & 93 & 4.5 & 0.20 & 0.14 & $\ldots$ & $\ldots$ & $9.53 \mathrm{e}-05$ & 2.17 & 0.14 \\
\hline $1921 \ldots \ldots$ & dS0 & 94 & 33.8 & 0.63 & 0.65 & $-2.98 \mathrm{e}-02$ & $2.96 \mathrm{e}-03$ & $7.74 \mathrm{e}-04$ & 0.55 & 0.12 \\
\hline $1936 \ldots . .$. & $\mathrm{dS} 0, \mathrm{~N}$ & 94 & 10.6 & 0.07 & 0.06 & $-4.75 \mathrm{e}-03$ & $2.92 \mathrm{e}-03$ & $1.49 \mathrm{e}-03$ & 1.06 & 0.17 \\
\hline $1942 \ldots \ldots$ & $\mathrm{dE}, \mathrm{N}$ & 93 & $\ldots$ & $0.34^{\mathrm{a}}$ & $\ldots$ & $\ldots$ & $\ldots$ & $\ldots$ & $\ldots$ & $\ldots$ \\
\hline $1948 \ldots \ldots$ & $\mathrm{dE}$ & 93 & 50.5 & 0.27 & 0.35 & $2.39 \mathrm{e}-02$ & $2.84 \mathrm{e}-03$ & $6.62 \mathrm{e}-03$ & 0.64 & 0.12 \\
\hline $1991 \ldots . .$. & $\mathrm{dE}, \mathrm{N}$ & 93 & 52.0 & 0.26 & 0.21 & $-9.06 \mathrm{e}-04$ & $3.34 \mathrm{e}-03$ & $3.44 \mathrm{e}-02$ & 1.04 & 0.15 \\
\hline $2004 \ldots \ldots$ & $\mathrm{dE}$ & 93 & 40.2 & 0.18 & 0.34 & $1.05 \mathrm{e}-02$ & $4.00 \mathrm{e}-03$ & $2.68 \mathrm{e}-02$ & 0.72 & 0.12 \\
\hline $2008 \ldots . .$. & $\mathrm{dE}$ & 93 & 85.5 & 0.57 & 0.54 & $8.01 \mathrm{e}-03$ & $1.52 \mathrm{e}-03$ & $3.24 \mathrm{e}-03$ & 1.06 & 0.03 \\
\hline $2019 \ldots \ldots$ & $\mathrm{dE}, \mathrm{N}$ & 94 & 28.5 & 0.26 & 0.24 & $7.35 \mathrm{e}-03$ & $1.29 \mathrm{e}-03$ & $1.01 \mathrm{e}-02$ & 0.64 & 0.13 \\
\hline $2042 \ldots \ldots$ & $\mathrm{dE}, \mathrm{N}$ & 93 & 36.6 & 0.10 & 0.12 & $-6.05 \mathrm{e}-03$ & $5.57 \mathrm{e}-03$ & $5.33 \mathrm{e}-03$ & 0.93 & 0.10 \\
\hline $2049 \ldots \ldots$ & $\mathrm{dE}, \mathrm{N}$ & 93 & 52.5 & 0.68 & 0.65 & $1.20 \mathrm{e}-02$ & $2.65 \mathrm{e}-03$ & $8.76 \mathrm{e}-04$ & 1.47 & 0.15 \\
\hline $2063 \ldots \ldots$ & $\mathrm{dE}$ & 93 & 21.0 & 0.24 & 0.38 & $-3.10 \mathrm{e}-02$ & $1.48 \mathrm{e}-02$ & $3.58 \mathrm{e}-02$ & 0.97 & 0.08 \\
\hline $2090 \ldots \ldots$ & $\mathrm{dE}, \mathrm{N}$ & 93 & 59.1 & 0.53 & 0.44 & $4.57 \mathrm{e}-03$ & $5.82 \mathrm{e}-03$ & $4.37 \mathrm{e}-02$ & 1.15 & 0.05 \\
\hline
\end{tabular}

${ }^{a}$ Mean ellipticity estimated as in Paper I.

When the fractional deviation $a_{4} / a<0$, the isophotes are called "boxy," and when $a_{4} / a>0$, the isophotes are called "disky." The sizes of the coefficients are, among other things, related to the fractional luminosity and orientation of embedded disks in spheroidal systems (e.g., Rix \& White 1990; Ryden 1992).

Typically the errors in these coefficients are large at the outer limits of the surface photometry, so in analogy with the definition $\langle\epsilon\rangle$, we define the intensity weighted boxiness coefficient $\left\langle a_{4} / a\right\rangle$ according to

$$
\left\langle a_{4} / a\right\rangle=\int\left(a_{4} / a\right) d L / \int d L .
$$

Columns 7 and 8 of Table 2 list the values of $\left\langle a_{4} / a\right\rangle$ for each galaxy and the error. The errors in $\left\langle a_{4} / a\right\rangle$, which we will denote as $\sigma\left(a_{4} / a\right)$, were estimated from the intensityweighted rms scatter about $\left\langle a_{4} / a\right\rangle$ as a function of semimajor axis $a$. In Figure 2, we show surface brightness contours of (top panel) VCC 1684, which has boxy contours $\left(100\left\langle a_{4} / a\right\rangle=-4.49 \pm 0.57\right)$, and of VCC 1334, which is disky $\left(100\left\langle a_{4} / a\right\rangle=-1.68 \pm 0.17\right)$. The contours on Figure 2 are at intervals of $0.5 \mathrm{mag}$ and show the images before star subtraction.

In Figure 4, we plot the mean boxiness $\left\langle a_{4} / a\right\rangle$ against the luminosity-weighted mean ellipticity $\langle\epsilon\rangle$. The top panel shows these parameters from our sample, where the symbols have the same meaning as in Figure 3. The error bar shows the mean uncertainty for the two quantities in our sample. The central and lower panels of Figure 4 display the same quantities for a samples of $\mathrm{E}$ and S0 galaxies as compiled by Peletier et al. (1990) and Bender et al. (1989), respectively. The circles and triangles in the lower two panels of Figure 4 represent, respectively, E and S0 galaxies. (Note that the authors of these two studies defined mean ellipticity and $a_{4} / a$ in a slightly different manner than we do here, and that there are several galaxies in common between their two studies.) The solid lines, which are the same in all three panels of this figure, show the envelope of the distribution in the plane of $\left(\langle\epsilon\rangle,\left\langle a_{4} / a\right\rangle\right)$ as drawn in Bender et al. (1989).

From Figure 4, we find that our combined sample of Virgo $\mathrm{dE}$ and dS0 galaxies shares some of the characteristics of the giant Es, in particular the same trend toward nearly elliptical isophotes $\left(\left\langle a_{4} / a\right\rangle=0\right)$ as the galaxies become rounder $(\langle\epsilon\rangle=0)$. This trend has been the basis of an argument (e.g., Bender et al. 1989; Kormendy \& Djorgovski 1989; Kormendy \& Bender 1996) that the majority of elliptical galaxies may have either boxy or disky isophotes, but appear round when viewed face-on because of projection effects (see Ryden 1992 for a longer discussion of the effects of projection). Furthermore, in Es there are correlations between departures from elliptical isophotes and the projected kinematics, which has led to the hypothesis (Kormendy \& Bender 1996) that there are two basic types of elliptical galaxies: those with significant rotational support (as seen through major-axis rotation curves), which have disky-distorted isophotes, and those with anisotropic velocity dispersions (as indicated by minor-axis rotation), which have boxy-distorted isophotes. This picture also includes data on presence or absence of cuspy inner brightness profiles (Kormendy \& Bender 1996 and references therein): the disky ellipticals are coreless while the boxy ellipticals have cuspy cores.

The extremely low surface brightness of the Virgo dEs and the low spatial resolution available from the ground mean that we naturally have no information on the cores or 

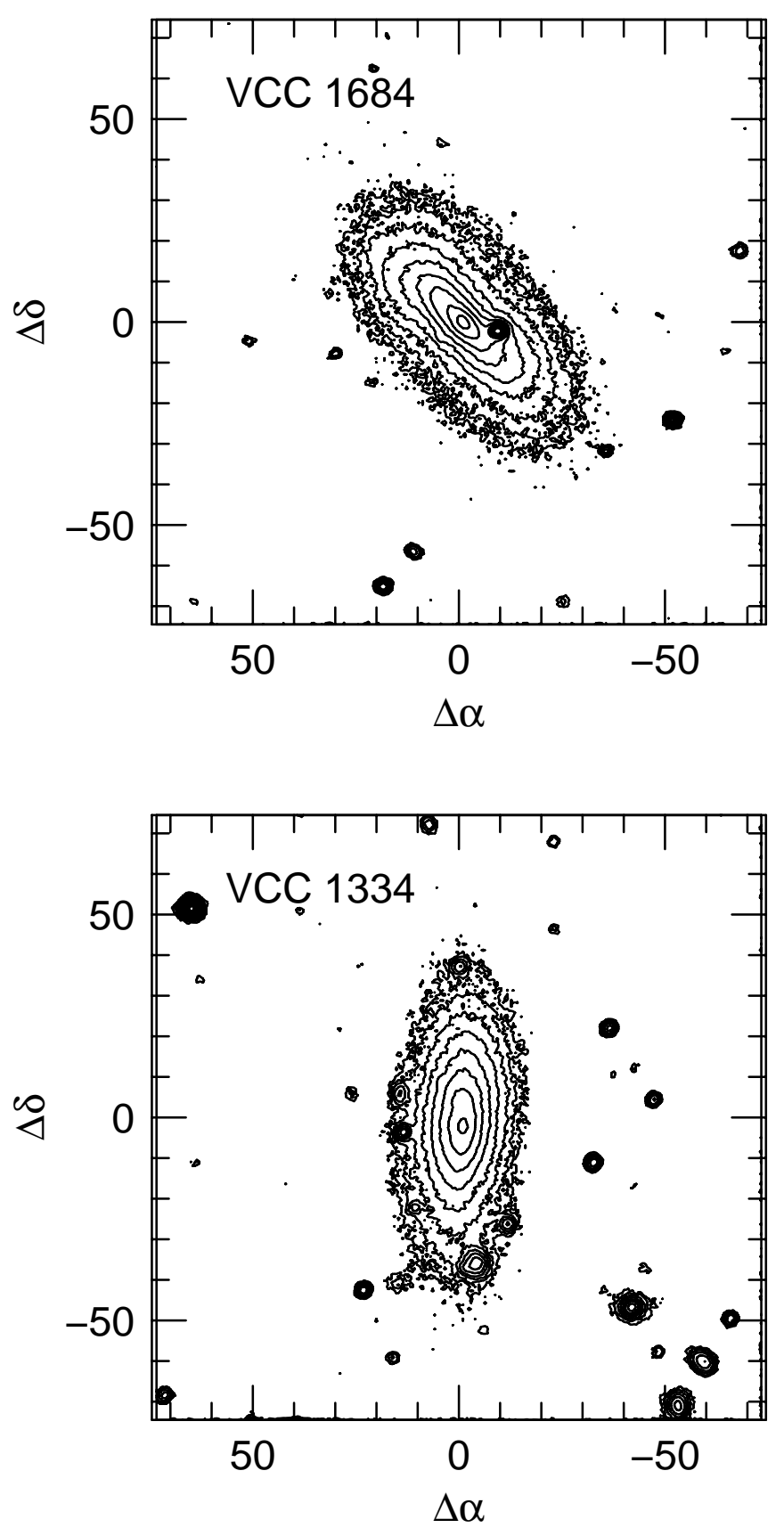

FIG. 2.-Boxy and disky profiles. Shown are isophotes for two dwarf galaxies that illustrate the range of $\left\langle a_{4} / a\right\rangle$ in our sample. Contour levels are at an interval of $0.5 \mathrm{mag}$. The galaxy in the upper panel has boxy isophotes, with $100\left\langle a_{4} / a\right\rangle=-4.49 \pm 0.57$, while the one in the lower panel has pointed (disky) isophotes, with $100\left\langle a_{4} / a\right\rangle=+1.68 \pm 0.17$. The images of stars that are superimposed on the galaxies were removed, as described in the text, before the surface brightness profiles were measured.

kinematics of the Virgo Cluster dEs. The near absence of round dEs that are boxy/disky and the increase in the range of $\left\langle a_{4} / a\right\rangle$ with increasing $\langle\epsilon\rangle$, both similar to the situation in $\mathrm{E}$ galaxies, suggests that future work on $\mathrm{dE}$ kinematics with very large telescopes may reveal two groups with differing rotational support.

In contrast with the more luminous E's, however, there are about a dozen galaxies in our sample that are flat $(\langle\epsilon\rangle>0.4)$ but which do not have boxy or disky isophotes. In other words, we do not see a significant gap in the distribution of $\left\langle a_{4} / a\right\rangle$ between boxy and disky galaxies at high

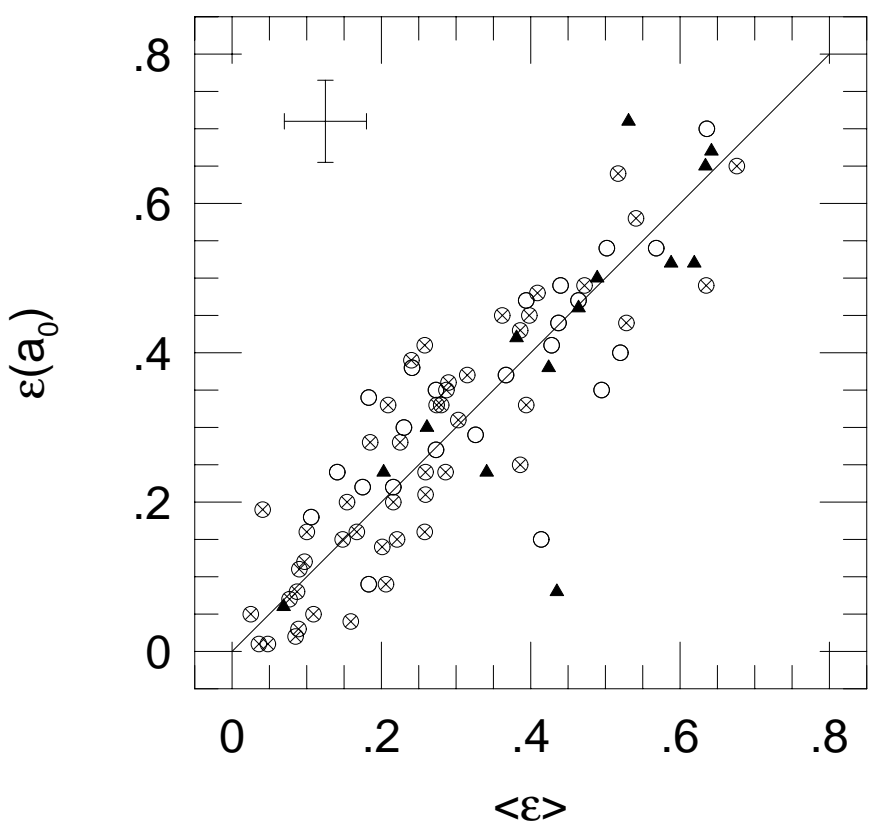

FIG. 3.-Comparison of the luminosity-weighted ellipticity $\langle\epsilon\rangle$ with the ellipticity of the isophote at the fiducial radius $\epsilon\left(a_{0}\right)$, as defined in the text. The solid line denotes unity. Open circles are for nonnucleated $\mathrm{dE}$ galaxies, while circles with a cross are for nucleated dEs. The filled triangles are for the dS0 galaxies.

ellipticity as claimed in the study by Bender et al. (1989). It is possible, of course, given the errors in the $\left\langle a_{4} / a\right\rangle$ values, that some of the points were scattered into the gap, but that would not account for all the points there. We therefore consider it possible that the dwarf galaxies differ from their larger cousins in that not all the highly flattened systems are either boxy or disky. Further study of highly flattened dwarf galaxies may confirm this result.

\subsection{Isophotal Twists}

We also defined a statistic $T$ to measure isophotal twists, which are common in elliptical galaxies (e.g., Jedrzejewski 1987; Nieto 1988; Kormendy \& Djorgovski 1989; Bender 1990). Consider two nested elliptical isophotes, the inner of which is at a surface brightness $\Sigma$ and has a semimajor axis $a$, axis ratio $q$, and position angle $\phi$. The outer isophote, at a surface brightness $\Sigma+d \Sigma$, has a semimajor axis $a+d a$, axis ratio $q+d q$, and position angle $\phi+d \phi$. By definition, $d a$ is positive, and generally $d \Sigma$ will be negative (luminosity decreases with radius). The values of $d q$ and $d \phi$ can have either sign.

One way to define the amount of twisting is to compute the power that must be added to the galaxy to "iron out" the twist. This approach has the advantage over simply measuring the change in the position angle with radius that it compensates for the large uncertainty in the position angle for very round galaxies. The luminosity-weighted mean position angle within the isophote with semimajor axis $a_{0}$ is

$$
\langle\phi\rangle=\frac{1}{L\left(a_{0}\right)} \int_{0}^{a_{0}} \phi(a) \Sigma(a) d A .
$$

Once we have the mean position angle $\langle\phi\rangle$, we can then calculate the twist $\gamma \equiv \phi(a)-\langle\phi\rangle$ for an isophote of semimajor axis $a$. The principal major axis thus intersects the 


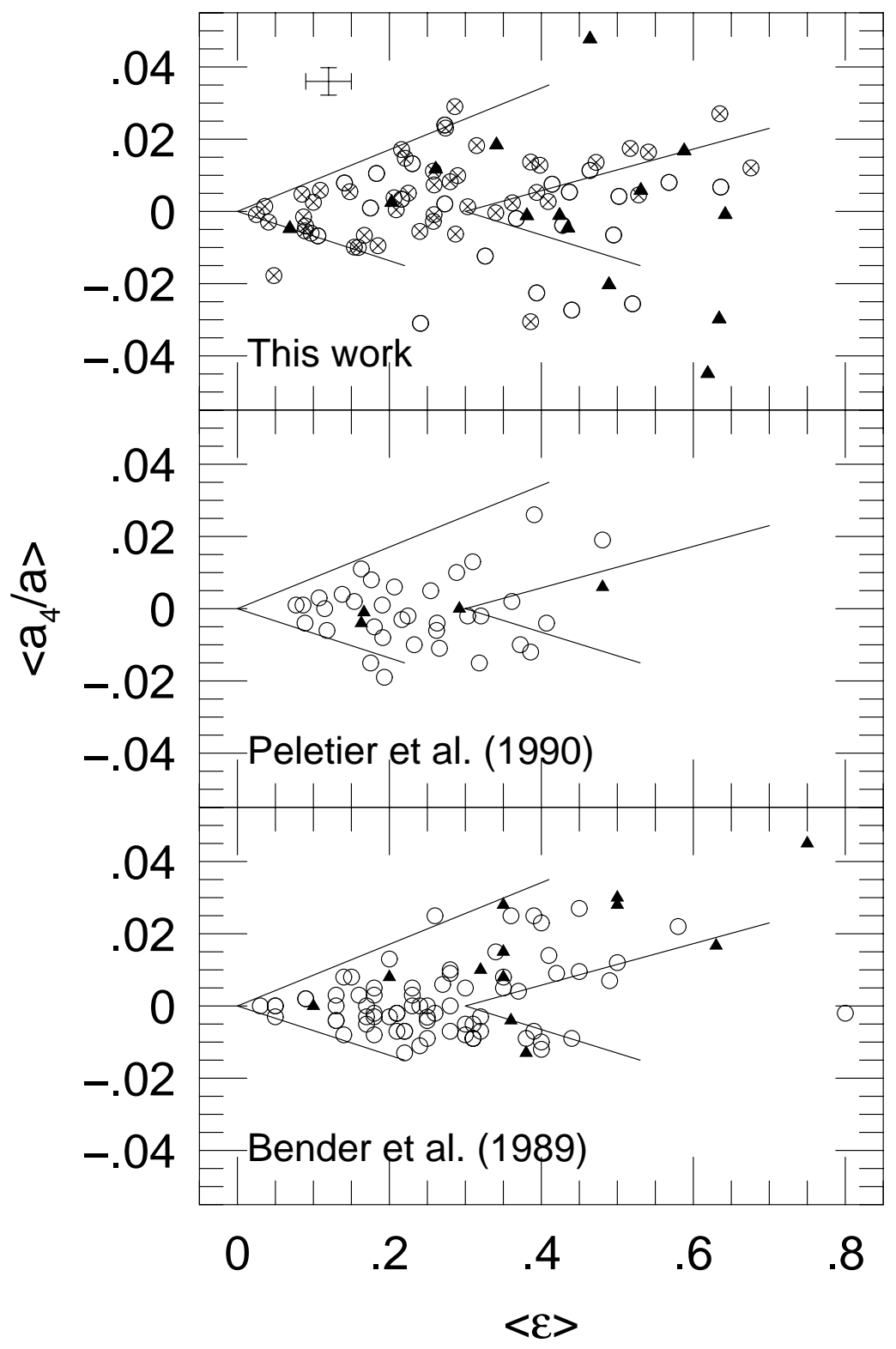

FIG. 4.-Mean departures from elliptical isophotes $\left\langle a_{4} / a\right\rangle$ against the ellipticity $\langle\epsilon\rangle$. The upper panel shows data from this paper, where the symbols have the same meaning as in Fig. 2. The error bar in this panel shows the mean errors in the two quantities. The central and lower panels show the equivalent data from the samples of giant ellipticals from Peletier et al. (1990) and from Bender et al. (1989), respectively. The solid lines, which are the same in all panels, show the approximate outer envelope of the distribution for the giant ellipticals as defined by Bender et al. In the lower two panels, Es are shown as open circles and S0s as filled triangles.

elliptical isophote at an angle $\gamma$ from the major axis of the isophote. The distance from the origin to the intersection of the principal major axis with the isophote is

$$
R=\frac{a}{\left(\sin ^{2} \gamma / q^{2}+\cos ^{2} \gamma\right)^{1 / 2}} .
$$

If we now twist the isophote to line up with the principal axes, the intensity around the ellipse will vary as $C \cos 2 \omega$, where $\omega$ is the angle with respect to the principal major axis. When we twist the isophote onto the principal major axis, however, its own major axis will intersect with lower surface brightness isophotes in the original galaxy. This is equivalent to sampling the surface brightness profile at a larger major axis distance, $a^{\prime}=a^{2} / R$, with $R$ given by equation (10). This gives $C=\Sigma\left(a^{\prime}\right)-\Sigma(a)$, or (doing a Taylor expansion)

$$
\begin{aligned}
C(a) & =\frac{d \Sigma}{d a} \frac{(a-R) a}{R}, \\
& =\frac{d \Sigma}{d a} a\left[\left(\frac{\sin ^{2} \gamma}{q^{2}+\cos ^{2} \gamma}\right)^{1 / 2}-1\right] .
\end{aligned}
$$

We are now ready to define the dimensionless twisting statistic $T$. After computing $\langle\phi\rangle$ for the light distribution within the limiting ellipse, we can then find $R(a)$ and $C(a)$ as a function of semimajor axis length. The statistic $T$ is defined as

$$
T\left(a_{0}\right)=\frac{2 \pi}{L\left(a_{0}\right)} \int_{0}^{a_{0}}|C(a)| a d a .
$$


The values of $T$ for each galaxy are listed in Column 9 of Table 2.

To illustrate the size of the twistiness statistic $T$, we display as Figure 5 contour plots and major-axis position angles of two galaxies with different twistiness. The contour levels are at the same interval as in Figure $2(0.5 \mathrm{mag})$. The top panel shows VCC 990, which has negligible isophotal twist $\left(T=3.4 \times 10^{-4}\right)$; the lower panel displays the brightness contours of VCC $1010\left(T=1.5 \times 10^{-2}\right)$, which has a major-axis twist of more than $15^{\circ}$ from the nucleus to the outermost isophotes.

To compare our values of $T$ for the Virgo dwarfs with those of more luminous E galaxies, we computed $T$ and $\langle\epsilon\rangle$
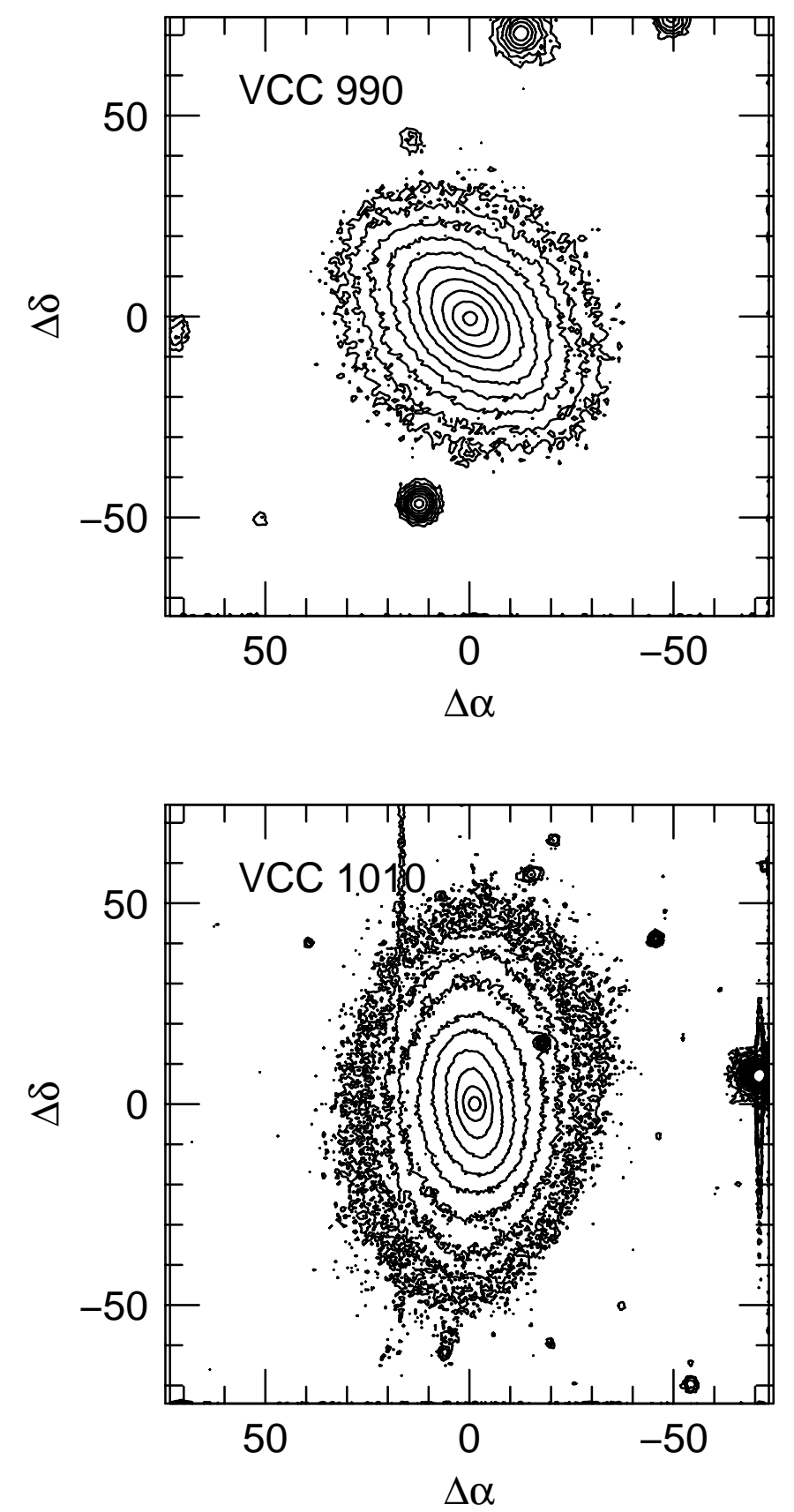

FIG. 5.-Examples of galaxies with and without twisted isophotes. The upper panel shows a galaxy with negligible isophotal twist, while the lower panel shows a galaxy with twisted isophotes. The latter galaxy has a value of the twistiness statistic, as defined in the text, of $T=0.015$. for 39 galaxies from Peletier et al. (1990); the values of $T$ are calculated readily from their listing of major-axis surface brightness and position angle, and are shown in Table 3. Figure 6 shows a plot of $T$ against $\langle\epsilon\rangle$ for our sample and that of Peletier et al., where the symbols have the same meaning as in Figures 3 and 4. There is evidently no correlation between ellipticity and the amount of isophotal twisting in either sample. The Virgo dwarfs do, however, have a significantly higher median $T$ than do the Peletier et al. Es. As measured by a Kolmogorov-Smirnoff test, the probability that the $\mathrm{dE}$ sample is drawn from the giant $\mathrm{E}$ sample is only $P_{\mathrm{KS}}=9.1 \times 10^{-8}$. We do not find any difference in the twistiness of nucleated and nonnucleated dEs.

The difference in the mean $T$ between dwarf and giant ellipticals is, however, correlated with galaxy luminosity. In Figure 7, we plot the values of $T$ for our sample and the Peletier et al. (1990) sample against the total blue absolute magnitude $M_{B}$. The brighter galaxies are from Peletier et al., and are shown as open triangles, while the fainter galaxies are from this paper using the same symbols as before. The absolute magnitudes for the giant Es were computed by Peletier et al., and are for a Hubble constant $H_{0}=50 \mathrm{~km}$

TABLE 3

\begin{tabular}{|c|c|c|c|}
\hline Name & $a_{0}(\operatorname{arcsec})$ & $\langle\epsilon\rangle$ & $T$ \\
\hline Abell $49 \ldots \ldots \ldots$ & 26.8 & 0.21 & $1.45 \mathrm{E}-02$ \\
\hline IC $1101 \ldots \ldots \ldots$ & 22.9 & 0.36 & $6.21 \mathrm{E}-04$ \\
\hline NGC $0315 \ldots . .$. & 55.6 & 0.26 & 3.19E-04 \\
\hline NGC $0720 \ldots \ldots$ & 85.6 & 0.41 & $1.11 \mathrm{E}-03$ \\
\hline NGC $0741 \ldots . .$. & 40.6 & 0.15 & $7.52 \mathrm{E}-04$ \\
\hline NGC $1052 \ldots .$. & 62.5 & 0.29 & $9.25 \mathrm{E}-04$ \\
\hline NGC $1129 \ldots . .$. & 64.7 & 0.19 & $5.65 \mathrm{E}-02$ \\
\hline NGC $1600 \ldots . .$. & 57.5 & 0.32 & $7.40 \mathrm{E}-04$ \\
\hline NGC $2300 \ldots . .$. & 56.2 & 0.16 & $4.03 \mathrm{E}-03$ \\
\hline NGC $2768 \ldots . .$. & 140.5 & 0.48 & $1.38 \mathrm{E}-03$ \\
\hline NGC $2778 \ldots . .$. & 27.1 & 0.22 & $2.13 \mathrm{E}-04$ \\
\hline NGC $2832 \ldots . .$. & 41.1 & 0.26 & $1.07 \mathrm{E}-03$ \\
\hline NGC $3377 \ldots . .$. & 72.7 & 0.48 & $2.71 \mathrm{E}-04$ \\
\hline NGC $3379 . . . .$. & 99.0 & 0.11 & $1.46 \mathrm{E}-04$ \\
\hline NGC $3605 . . . .$. & 25.5 & 0.39 & $2.26 \mathrm{E}-04$ \\
\hline NGC $3665 \ldots \ldots$ & 66.2 & 0.22 & $8.58 \mathrm{E}-04$ \\
\hline NGC $3801 \ldots . .$. & 39.0 & 0.33 & $1.20 \mathrm{E}-03$ \\
\hline NGC $4261 \ldots . .$. & 72.9 & 0.19 & 8.87E-05 \\
\hline NGC $4278 \ldots . .$. & 66.8 & 0.11 & $4.35 \mathrm{E}-03$ \\
\hline NGC $4374 \ldots . .$. & 107.0 & 0.12 & $1.03 \mathrm{E}-03$ \\
\hline NGC $4387 \ldots . .$. & 33.1 & 0.37 & $6.32 \mathrm{E}-04$ \\
\hline NGC $4406 \ldots . .$. & 141.9 & 0.23 & $1.47 \mathrm{E}-03$ \\
\hline NGC $4472 \ldots . .$. & 161.8 & 0.16 & $9.78 \mathrm{E}-04$ \\
\hline NGC $4478 \ldots . .$. & 34.7 & 0.18 & $2.84 \mathrm{E}-03$ \\
\hline NGC $4486 \ldots . .$. & 144.1 & 0.08 & $1.81 \mathrm{E}-03$ \\
\hline NGC $4551 \ldots . .$. & 33.5 & 0.27 & 4.57E-05 \\
\hline NGC $4636 \ldots . .$. & 126.6 & 0.17 & $2.63 \mathrm{E}-03$ \\
\hline NGC $4649 \ldots . .$. & 137.7 & 0.18 & $5.31 \mathrm{E}-04$ \\
\hline NGC $4697 \ldots . .$. & 117.1 & 0.39 & $8.69 \mathrm{E}-05$ \\
\hline NGC $4874 \ldots \ldots$ & 42.6 & 0.09 & 8.96E-04 \\
\hline NGC $4889 \ldots . .$. & 45.8 & 0.32 & 4.54E-04 \\
\hline NGC $5638 \ldots . .$. & 42.8 & 0.09 & 7.13E-04 \\
\hline NGC $5813 \ldots . .$. & 74.4 & 0.19 & $2.30 \mathrm{E}-03$ \\
\hline NGC $5831 \ldots \ldots$ & 39.8 & 0.18 & $1.10 \mathrm{E}-02$ \\
\hline NGC $5845 \ldots . .$. & 20.2 & 0.31 & $1.34 \mathrm{E}-03$ \\
\hline NGC $6051 \ldots . .$. & 31.3 & 0.30 & $5.95 \mathrm{E}-04$ \\
\hline NGC $6086 \ldots . .$. & 17.8 & 0.29 & $2.30 \mathrm{E}-04$ \\
\hline NGC $6269 . . . .$. & 29.0 & 0.25 & $3.01 \mathrm{E}-04$ \\
\hline NGC $7626 \ldots . .$. & 49.5 & 0.14 & $8.71 \mathrm{E}-04$ \\
\hline
\end{tabular}




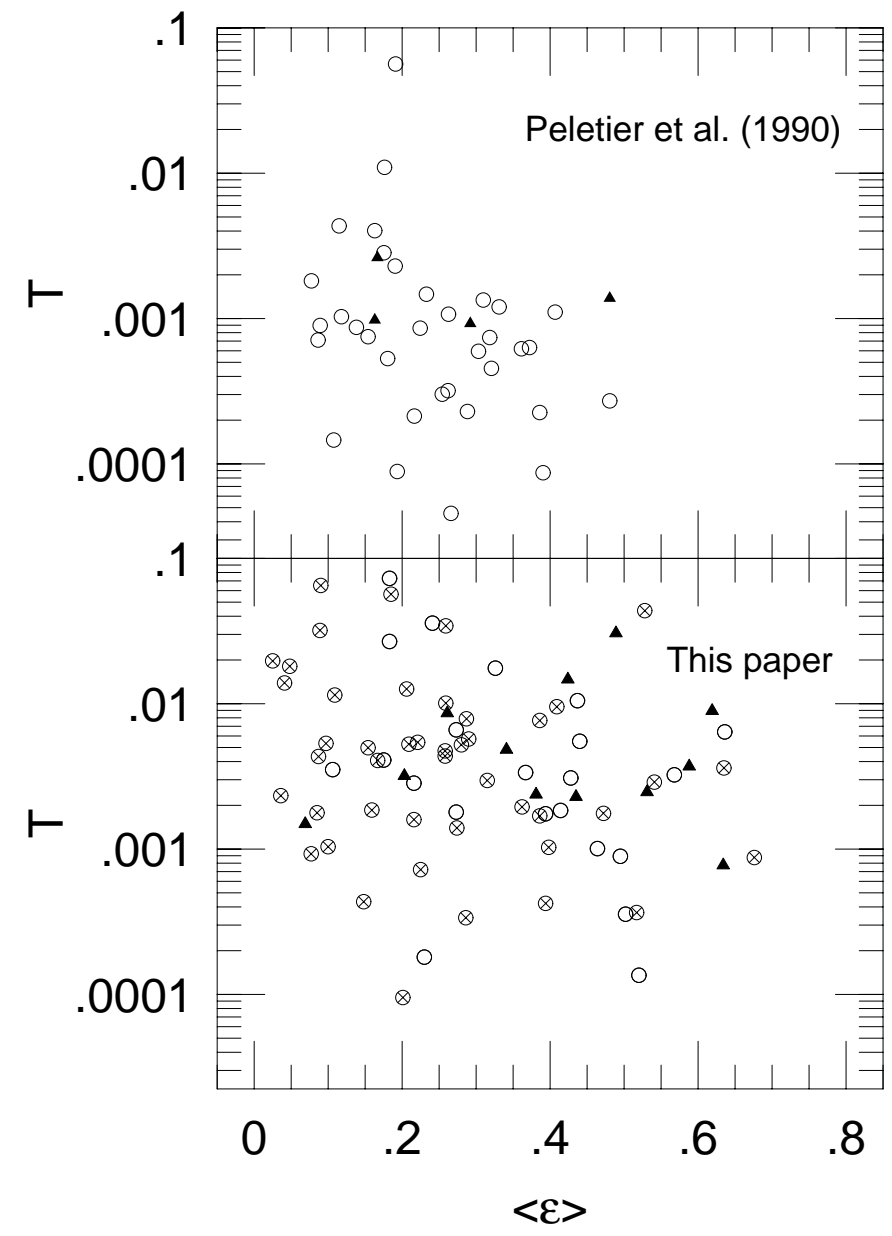

FIG. 6.-Correlation of isophotal twists and ellipticity. The lower panel shows $T$ against $\langle\epsilon\rangle$ for our sample, where the symbols are the same as in Fig. 3. The upper shows the same quantities for the Peletier et al. (1990) study of giant $\mathrm{E}$ galaxies, measured as described in the text.

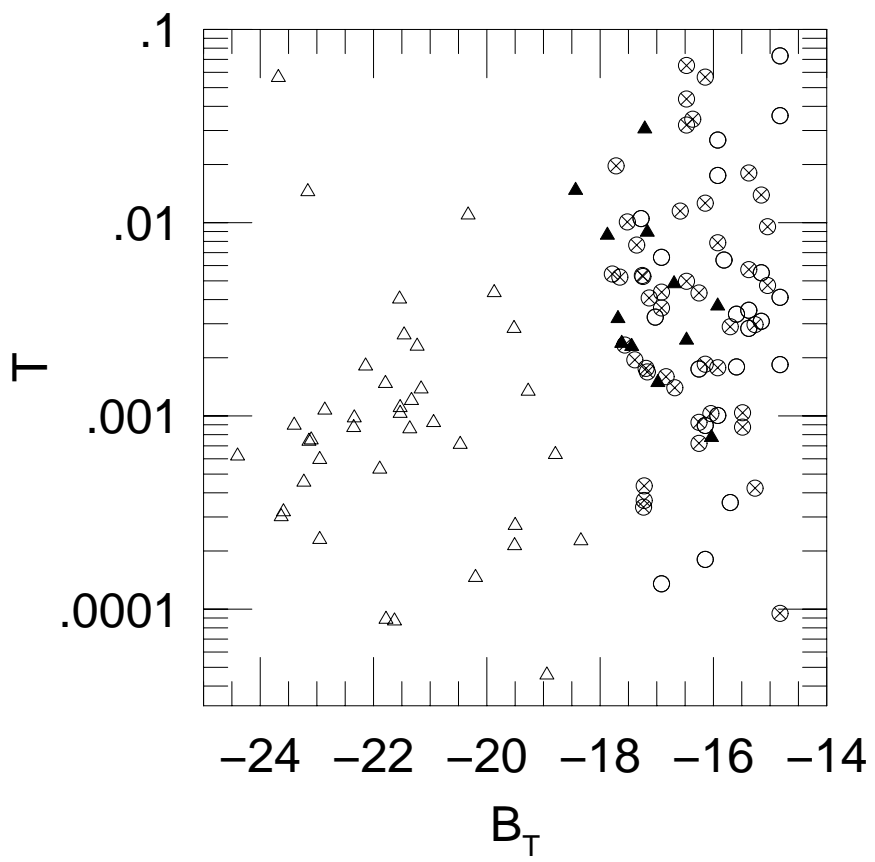

Fig. 7.- Measure of isophotal twists against $B$-band absolute magnitude. Open triangles are from Peletier et al. (1990), while other symbols are from this study and have the same meaning as in Fig. 3. Absolute magnitudes were computed as described in the text. $\mathrm{s}^{-1} \mathrm{Mpc}^{-1}$. We computed $M_{B}$ for the dEs using the total blue magnitude $B_{T}$ in the VCC for a distance modulus to Virgo of 31.75, which is consistent with values of $H_{0}$ near 50 $\mathrm{km} \mathrm{s}^{-1} \mathrm{Mpc}^{-1}$ (in any case, the particular choice of $H_{0}$ does not matter here). Though there is a wide scatter in $T$ at any magnitude, there is a general trend toward smaller values of isophotal twists with increasing galaxy luminosity.

\subsection{Shapes of the Major Axis Profile}

Finally, we fit the major-axis brightness profiles of each galaxy according to the power-law formula

$$
\Sigma(a) \propto \exp \left[-\left(a / a_{s}\right)^{n}\right],
$$

where $a_{s}$ is a scale radius for the profile and $n$ is the exponent variable. When $n=1$, the profile is exponential, while when $n=1 / 4$ the profile is represented by a de Vaucouleurs (1948) function. This function was originally introduced by Sérsic (1968); it has been revived recently in the studies of the structure in the bulges of spiral galaxies (e.g., Andredakis, Peletier \& Balcells 1995; Courteau, de Jong \& Broeils 1996) and has been measured for a number of dwarf galaxies in the Virgo Cluster, as we will discuss shortly. We performed the fit from an interior radius of $a_{i}=5^{\prime \prime}$ out to the radius $a_{0}$ defined above. The values of $n$ and the error $\sigma(n)$ are listed in Columns 10 and 11 of Table 2. The error estimate derived by remeasuring the profile with the sky level adjusted by $\pm 1 \sigma$, where $\sigma$ is the measured error in the sky.

Several recent papers on Virgo dEs have discussed measures of the Sérsic parameter $n$. Jerjen \& Binggeli (1997) have shown a continuous trend from de Vaucouleurs profiles toward exponential profiles with decreasing luminosity in Virgo Es/dEs; they therefore argued that dEs are a lowluminosity extension of classical, bright ellipticals. Young \& Currie $(1994,1995)$ found a relatively tight relationship between $n$ and the total magnitude for Fornax cluster dEs; they then went on to find a much larger scatter in Virgo dEs and claimed that this scatter must be caused by a great depth of the Virgo Cluster along the line of sight. This conclusion was discussed and extensively criticized by Binggeli \& Jerjen (1998). These three studies employed photographic plate material in the measurement of the surface brightness profiles. Recently, Durrell (1997) presented Washington CCD-based photometry of a small number of Virgo dEs and tabulated the Sérsic parameter $n$ for his sample.

In Figure 8 we compare our values of $n$ with those previously measured for Virgo dEs, where the comparisons are against (top to bottom) Durrell (1997), Young \& Currie 1995, and Binggeli \& Jerjen (1998). Our values are tightly correlated with those of both Young \& Currie 1995 and Binggeli \& Jerjen (1998) (though with a few discrepant points), but there is a significant offset between our determination and that of these two studies. We are unable to determine the source of this offset (the other papers discuss that the determination of $n$ is rather sensitive to the choice of inner and outer radii over which the profile is fit). Given that the offsets in each case are about the same size as our errors in $n$ that are dominated errors in the measurement of the sky level, it may also be that there are systematic differences in how the sky is found on our CCD data versus the photographic data in these studies. Our values are in statistical agreement with those in Durrell (1997), though the range of $n$ for galaxies in common does not extend to high enough values 


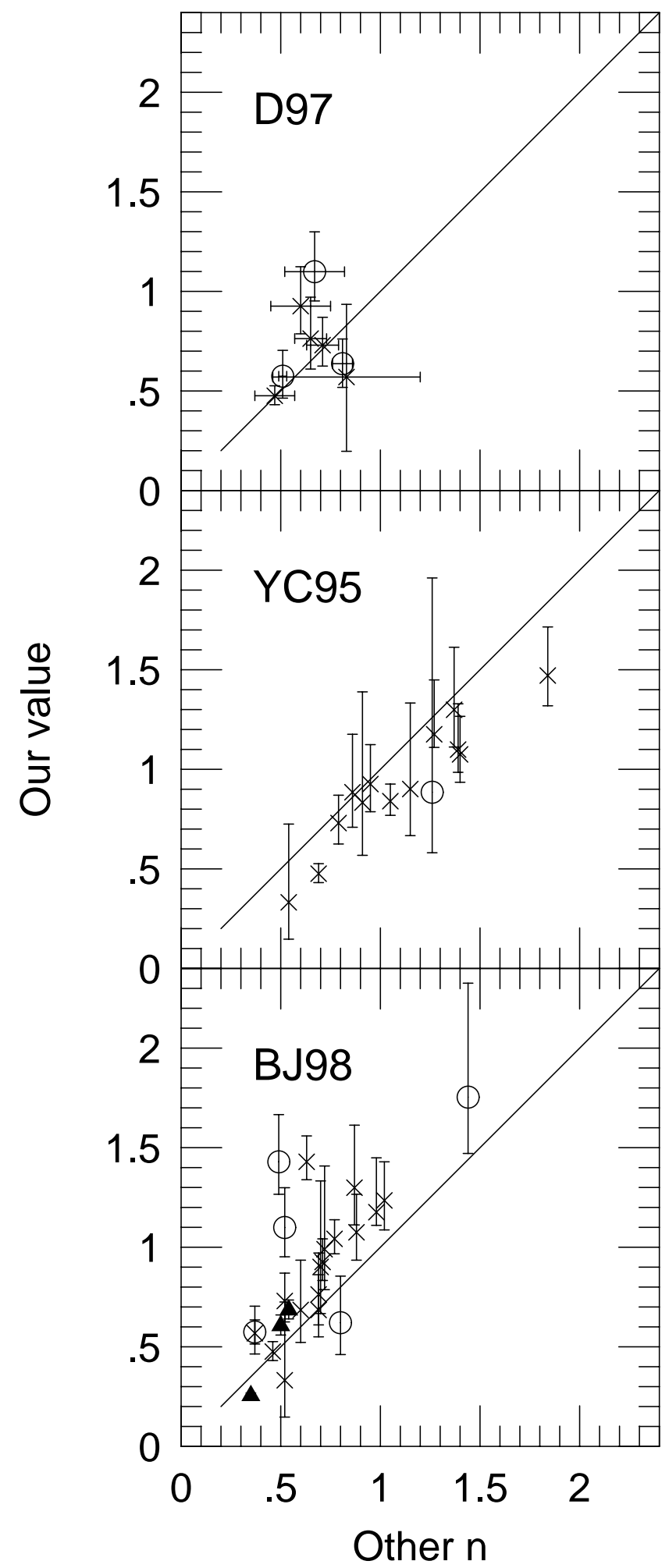

FIG. 8.-Comparison of our values of the major-axis shape with previously published values. The top, central, and lower panels show galaxies in common with Durrell (1997), Young \& Currie (1995), and Binggeli \& Jerjen (1998). Solid lines denote identity.

to determine whether there may be a systematic difference between the two samples.

Figure 9 displays the correlation between $n$ and total blue magnitude for our sample. We confirm the previous results of Young \& Currie 1995, Jerjen \& Binggeli (1997), Binggeli

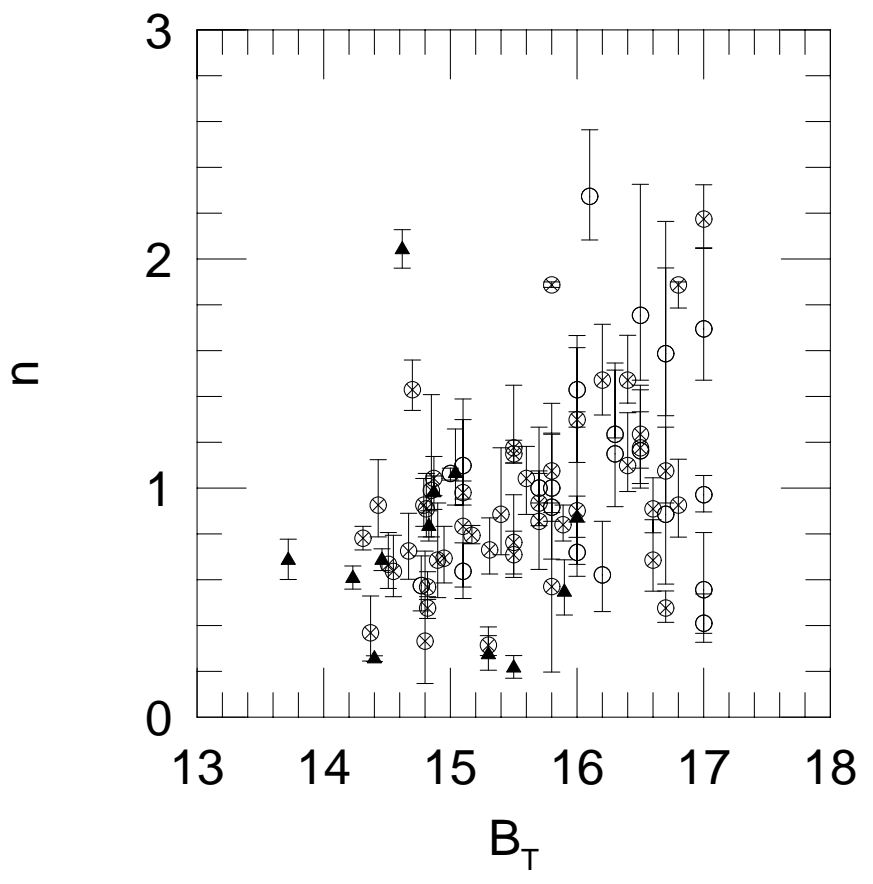

FIG. 9.-Distribution of the Sérsic (1968) parameter $n$ for the majoraxis profile against total blue magnitude for our sample. Symbols are as in Fig. 3. Error bars show the effect of errors in the determination of the sky level.

\& Jerjen (1998) and Paper I that dEs in Virgo have profiles that are close to exponential, with lower values of $n$ as the galaxy luminosity increases.

\section{SUMMARY AND DISCUSSION}

We have obtained new surface photometry of several more dEs and most of the dwarf galaxies that are designated "dSO" in the VCC, and have measured a set of parameters to describe the surface photometry of these in combination with images from our earlier study of $70 \mathrm{dE}$ galaxies (Paper I). Our parameters were the intensityweighted mean ellipticity and departure from elliptical isophotes, the power-law slope of the major-axis profile, and a newly defined statistic to measure isophotal twists.

Because the dE galaxies in our sample show overall similarity in the distribution of $\left\langle a_{4} / a\right\rangle$ with $\langle\epsilon\rangle$ to the giant systems, we suggest that the dwarf galaxies may have the same dichotomy between rotating and nonisotropic systems that is seen in the more luminous systems. As we showed in Paper I, most of the galaxies in our sample are fainter than the sky even in their centers, so obtaining confirmatory kinematic information for the low surface-brightness dwarf galaxies in the Virgo Cluster would be extremely difficult. Our photometry does not have the kind of spatial resolution (subarcsecond) to detect cuspy cores.

We also find that, contrary to our assertion in Paper I, the Virgo dEs have significantly larger isophotal twists, on average, than giant Es. Since we took our E galaxy sample from Peletier et al. (1990), which is mainly composed of field ellipticals, there exists the possibility that the degree of isophotal twisting depends on environment and that a study of luminous $\mathrm{E}$ galaxies in Virgo or other clusters of comparable richness may yield a different result. The main results from this work and Paper I are that dEs/dSOs have a flatter 
distribution of ellipticities, more exponential profiles, and a greater amount of isophotal twist than larger ellipticals.

Can we conclude that $\mathrm{dE} / \mathrm{dS} 0$ galaxies form a different class of object than larger Es? This depends primarily on whether there is a statistically significant break with magnitude in the properties of ellipticals between dwarfs and giants as has been suggested, for example, by Tremblay \& Merritt (1996). Our analysis of the Virgo dEs does not shed light on this issue. As Young \& Currie (1995) found, we see a slow trend with galaxy luminosity toward more exponential profiles, and the trends in isophotal twists (Figure 7) are similar. Furthermore, the analysis of the departures of isophotes from pure ellipses (Figure 4) shows that the dEs share the same basic characteristics as large Es: round galaxies are almost always elliptical, but flattened galaxies can be either boxy or disky.

What, if anything, are dS0 galaxies? We do not find that any of our parameters that describe the surface photometry are correlatives with the $\mathrm{dSO} / \mathrm{dE}$ designation. Sandage \& Binggeli (1984) introduced the class dS0 galaxies with the cautious declaration, "This is a new class, if it indeed exists." Like dE galaxies, dS0 galaxies have low central surface brightness and typically exponential profiles. In the classification scheme of Sandage \& Binggeli, dS0 galaxies are photometrically distinguished from $\mathrm{dE}$ galaxies either by direct evidence of a disk (revealed by disky isophotes in an edge-on dwarf) or by evidence for the transition from a central bulge to an outer disk (revealed by a change in slope of the radial light distribution). The only way in which the
dS0 galaxies in the VCC seem different from the dE galaxies is that they may represent the tail of the distribution toward flatter shapes and/or larger values of the profile exponent $n$ (the latter indicates shallower outer profiles than near the center). These, however, were two of the original criteria for calling them dS0s in the first place (Binggeli et al. 1985; Binggeli \& Popescu 1995), and in any case the dS0s are well mixed with the dEs in any of our plots. Because our data are not photometrically calibrated, we cannot measure the possibility that the dSOs separate from dEs in (for example) a comparison of central surface brightness and scale length, as might be expected if they have a strong bulge/disk substructure. This has been addressed in other studies, for example in Binggeli \& Jerjen (1998) (especially their Figure 6), and no difference is found. The dS0 galaxies do not, in general, have disky isophotes or a bulge/disk structure as represented by a change in ellipticity with radius. We therefore conclude that, although there may be individual systems that share some characteristics of larger S0s, there are no compelling arguments from the analysis of their surface photometry for a separate class of dS0 galaxies.

We would like to thank the staff at the Lowell Observatory for their assistance during our observations. We acknowledge support from the National Science Foundation grants AST-9157038 to D. T., AST-9112897 to R. P., and AST-9357396 to B. R. Thanks also to Tom Statler and to the referee for valuable suggestions.

\section{REFERENCES}

Andredakis, Y. C., Peletier, R. F., \& Balcells, M. 1995, MNRAS, 275, 874

Bender, R. 1990, in Dynamics and Interactions of Galaxies, ed. R. Wielen (Berlin: Springer), 232

Bender, R., Surma, P., Döbereiner, S., Möllenhoff, C., \& Madejsky, R. 1989, A\&A, 217, 35

Binggeli, B., \& Cameron, L. M. 1991, A\&A, 252, 27

Binggeli, B., \& Jerjen, H. 1998, A\&A, 333, 17

Binggeli, B., \& Popescu, C. C. 1995, A\&A, 298, 73

Binggeli, B., Sandage, A., \& Tammann, G. A. 1985, AJ, 90, 1681

Binggeli, B., Sandage, A., \& Tarenghi, M. 1984, AJ, 89, 64

Caldwell, N. 1983, AJ, 88, 804

Carter, D. 1978, MNRAS, 212, 767

Courteau, S., de Jong, R. S., \& Broeils, A. H. 1996, ApJ, 457, L73

de Vaucouleurs, G. 1948, Ann. d'Astrophys., 11, 247

Durrell, P. R. 1997, AJ, 113, 531

Ichikawa, S.-I., Wakamatsu, K.-I., \& Okamura, S. 1986, ApJS, 60, 475

Impey, C., Bothun, G., \& Malin, D. 1988, ApJ, 330, 634

James, P. 1991, MNRAS, 250, 544

Jedrzejewski, R. I. 1987, in IAU Symp. 127, Structure and Dynamics of Elliptical Galaxies, ed. T. de Zeeuw (Dordrecht: Reidel), 257

Jerjen, H., \& Binggeli, B. 1997, in ASP. Conf. Ser. 116, The Nature of Elliptical Galaxies, ed. G. S. DaCosta \& P. Saha (San Francisco: ASP), 239
Kormendy, J., \& Bender, R. 1996, ApJ, 464, L119

Kormendy, J., \& Djorgovski, S. 1989, ARA\&A, 27, 235

Lauer, T. R. 1985, ApJS, 57, 473

Nieto, J.-L. 1988, in Segunda Reunion Regional Sobre Astronomia Extragalactica, ed. J. Sersic et al. (Cordoba: Academia Nacional de Ciencias), 239

Peletier, R. F., Davies, R. L., Illingworth, G. D., Davies, L. E., \& Cawson, M. 1990, AJ, 100, 1091

Pogge, R. W., Atwood, B., Byard, P. L., O’Brien, T. P., Peterson, B. M., Lame, N. J., \& Baldwin, J. A. 1995, PASP, 107, 1226

Reaves, G. 1956, AJ, 61, 69

1983, ApJS, 53, 575

Rix, H.-W., \& White, S. D. M. 1990, ApJ, 362, 52980

Ryden, B. S. 1992, ApJ, 386, 42

Ryden, B. S., \& Terndrup, D. M. 1994, ApJ, 425, 43 (Paper I)

Sandage, A., \& Binggeli, B. 1984, AJ, 89, 919

Sérsic, J. L. 19 , Atlas de galaxias australes (Cordoba: Observatorio Astronomico)

Stetson, P. B. 1987, PASP, 99, 191

Tremblay, B., \& Merritt, D. 1996, AJ, 111, 2243

Young, C. K., \& Currie, M. J. 1994, MNRAS, 268, L11

. 1995, MNRAS, 273, 1141 